\title{
SPECTRUM OF A LINEARIZED AMPLITUDE EQUATION FOR ALTERNANS IN A CARDIAC FIBER*
}

\author{
SHU DAI ${ }^{\dagger}$ AND DAVID G. SCHAEFFER ${ }^{\dagger}$
}

\begin{abstract}
Under rapid periodic pacing, cardiac cells typically undergo a period-doubling bifurcation in which action potentials of short and long duration alternate with one another. If these action potentials propagate in a fiber, the short-long alternation may suffer reversals of phase at various points along the fiber, a phenomenon called (spatially) discordant alternans. Either stationary or moving patterns are possible. Using a weak approximation, Echebarria and Karma proposed an equation to describe the spatiotemporal dynamics of small-amplitude alternans in a class of simple cardiac models, and they showed that an instability in this equation predicts the spontaneous formation of discordant alternans. To study the bifurcation, they computed the spectrum of the relevant linearized operator numerically, supplemented with partial analytical results. In the present paper we calculate this spectrum with purely analytical methods in two cases where a small parameter may be exploited: (i) small dispersion or (ii) a long fiber. From this analysis we estimate the parameter ranges in which the phase reversals of discordant alternans are stationary or moving.
\end{abstract}

Key words. spectrum, amplitude equation, cardiac alternans

AMS subject classifications. 35B32, 92C30

1. Introduction. Alternans, a period-doubling bifurcation of action potential durations in rapidly paced cardiac cells, has been implicated as a precursor of ventricular fibrillation $[1,2,3,4]$. When such action potentials propagate in tissue, their short-long alternation may suffer reversals of phase; such discordant alternans pose even higher arrythmogenic risks. Since ventricular fibrillation accounts for $1 / 6$ of all deaths in the US $[9,10]$, great importance attaches to understanding these phenomena.

Echebarria and Karma [5] proposed a weakly nonlinear description of the onedimensional evolution of discordant alternans in cardiac models ${ }^{1}$ for which each action potential duration (APD) is a function of only the previous diastolic interval (DI). To set the context, suppose a cardiac fiber of length $L$ is stimulated periodically at its $x=0$ end, say with period $B$ (mnemonic for basic cycle length, which has the acronym BCL). It is assumed that each stimulus successfully generates an action potential that propagates down the fiber. Let $A_{k}(x)$ be the duration of the $k^{t h}$ action potential at the position $x$ along the fiber. For slow stimulation, say $B>B_{\text {crit }}$, the propagating action potentials become identical after a transient: i.e., $\lim _{k \rightarrow \infty} A_{k}(x)$ exists and is independent of $x$. In studying pacing with $B<B_{\text {crit }}$, Echebarria and Karma make the ansatz

$$
A_{k}(x)=A_{\text {crit }}-\delta A+(-1)^{k} a(x, t),
$$

where $A_{\text {crit }}$ is the APD when pacing with period $B=B_{\text {crit }}, \delta A$ is the average shortening of APD resulting from decreasing $B$ below $B_{\text {crit }}$, and $a(x, t)$ is the amplitude of alternans, assumed slowly varying. Because of this slow-variation assumption, one may study the evolution of $a$ with respect to a continuous time $t$ that interpolates

\footnotetext{
${ }^{*}$ This work was supported by National Institutes of Health under grant 1R01-HL-72831 and the National Science Foundation under grant PHY-0549259.

${ }^{\dagger}$ Department of Mathematics and Center for Nonlinear and Complex Systems, Duke University, Durham, NC 27708 USA.

${ }^{1}$ No cardiology background is required to read this paper if equation (1.2) is accepted as a given. An appendix, written primarily for mathematicians, reviews the context in which (1.2) arises.
} 
between the times $t=k B, k=0,1,2, \ldots$, when stimuli are applied. Nondimensionalizing the time by $B_{\text {crit }}$, they derive the evolution equation for $a(x, t)$

$$
\partial_{t} a=\sigma a+\xi^{2} \partial_{x x} a-w \partial_{x} a-\frac{1}{\Lambda} \int_{0}^{x} a\left(x^{\prime}, t\right) d x^{\prime}-g a^{3}
$$

where $\sigma$ is the bifurcation parameter, which is dimensionless and proportional to $B_{\text {crit }}-B ; \Lambda, w, \xi$ are positive parameters, each having the units of length, that are derived from the equations of the cardiac model; and the nonlinear term $-g a^{3}$ limits growth after the onset of linear instability. Boundary conditions

$$
\partial_{x} a(0, t)=0, \quad \partial_{x} a(L, t)=0
$$

are imposed on (1.2).

Of course $a \equiv 0$ is a solution of (1.2-1.3), but it loses stability as $\sigma$ increases. Bifurcation analysis of this equation requires knowing the eigenvalues $\Omega_{n}$ of the linear operator that maps a function $a(x)$ to

$$
\xi^{2} \partial_{x x} a-w \partial_{x} a-\frac{1}{\Lambda} \int_{0}^{x} a\left(x^{\prime}, t\right) d x^{\prime}
$$

subject to Neumann boundary conditions. All of these eigenvalues lie in the (stable) left-half plane. The eigenvalue(s) with the largest real part, say $\Omega_{\max }$, determines the character of the solution of (1.2) at the onset of bifurcation - a stationary pattern if $\Omega_{\max }$ is real, a moving pattern if it is complex.

In this paper we extend the results of [5] by calculating the spectrum of (1.4) with purely analytical means in two limiting cases: small dispersion and a long fiber. In particular, it follows from our analysis that in a long fiber $\Omega_{\max }$ is real if, modulo terms that are $\mathcal{O}\left(L^{-2}\right)$,

$$
\Lambda^{-1} \leq C \frac{w^{3}}{\xi^{4}}
$$

where

$$
C=\frac{1}{64}(71+17 \sqrt{17}) \approx 2.205
$$

and $\Omega_{\max }$ is complex otherwise.

2. The Eigenvalue Problem. Let us begin by nondimensionalizing (1.4). The parameters $\xi, w, \Lambda$, like $L$, all have the units of length. Thus we define a set of new parameters

$$
\bar{w}=w / \xi, \quad \bar{\Lambda}=\Lambda / \xi, \quad \bar{L}=L / \xi
$$

and (1.4) can be written as

$$
\frac{d^{2} a}{d \bar{x}^{2}}-\bar{w} \frac{d a}{d \bar{x}}-\bar{\Lambda}^{-1} \int_{0}^{\bar{x}} a\left(\bar{x}^{\prime}\right) d \bar{x}^{\prime}
$$

where $\bar{x}=x / \xi$. For further scaling, we define

$$
\overline{\bar{x}}=\bar{w} \cdot \bar{x}, \quad \overline{\bar{L}}=\bar{w} \cdot \bar{L}, \quad \overline{\bar{\Lambda}}=\bar{\Lambda} \cdot \bar{w}^{3}
$$


and an operator

$$
\mathscr{L}=\frac{d^{2} a}{d \overline{\bar{x}}^{2}}-\frac{d a}{d \overline{\bar{x}}}-\overline{\bar{\Lambda}}^{-1} \int_{0}^{\overline{\bar{x}}} a\left(\overline{\bar{x}}^{\prime}\right) d \overline{\bar{x}}^{\prime}
$$

One observes that (1.4) equals $\bar{w}^{2} \cdot \mathscr{L} a$.

The analysis in Sections 2-4 below uses dimensionless variables, but we nonetheless shall omit all the bars in (2.4). Suppose $a(x)$ is an eigenfunction of (2.4) with eigenvalue $\Omega$ : i.e.

$$
\mathscr{L} a=\Omega a, \quad \text { with } \quad \mathrm{a}^{\prime}(0)=0, \quad \mathrm{a}^{\prime}(\mathrm{L})=0 .
$$

To eliminate the integral term in (2.4), we differentiate this equation (but not the B.C.) with respect to $x$ to obtain

$$
\left\{\begin{array}{l}
a^{\prime \prime \prime}-a^{\prime \prime}-\Lambda^{-1} a=\Omega a^{\prime} \\
a^{\prime}(0)=0 \\
a^{\prime}(L)=0 \\
a^{\prime \prime}(0)=\Omega a(0) .
\end{array}\right.
$$

The additional B.C. comes from evaluating the eigenvalue equation, before differentiation, at $x=0$. A function of the form $a(x)=e^{\kappa x}$ satisfies the ODE in (2.6) if

$$
\kappa^{3}-\kappa^{2}-\Omega \kappa-\Lambda^{-1}=0 .
$$

If $\kappa_{1}, \kappa_{2}, \kappa_{3}$ are the roots of (2.7), then this equation may be reformulated as

$$
\begin{aligned}
& 1=\kappa_{1}+\kappa_{2}+\kappa_{3}, \\
& \Omega=-\left(\kappa_{1} \kappa_{2}+\kappa_{2} \kappa_{3}+\kappa_{3} \kappa_{1}\right), \\
& \Lambda^{-1}=\kappa_{1} \kappa_{2} \kappa_{3} .
\end{aligned}
$$

Assuming the roots $\kappa_{1}, \kappa_{2}, \kappa_{3}$ are distinct, we seek a solution of (2.6) of the form $a(x)=\sum_{1}^{3} C_{i} e^{\kappa_{i} x}$. The three B.C.s in (2.6) give a homogeneous linear system for the unknown coefficients $C_{i}$. For this system to possess a nontrivial solution, we need

$$
\operatorname{det}\left(\begin{array}{ccc}
\kappa_{1} & \kappa_{2} & \kappa_{3} \\
\kappa_{1} e^{\kappa_{1} L} & \kappa_{2} e^{\kappa_{2} L} & \kappa_{3} e^{\kappa_{3} L} \\
\Omega-\kappa_{1}^{2} & \Omega-\kappa_{2}^{2} & \Omega-\kappa_{3}^{2}
\end{array}\right)=0 .
$$

Thus $\Omega \in \mathbb{C}$ is an eigenvalue of $\mathscr{L}$ if there exist a triple $\kappa_{1}, \kappa_{2}, \kappa_{3}$, no two of them equal, such that the four equations (2.8-2.11) are satisfied.

If $\Lambda^{-1}>0$ so that each root $\kappa_{i}$ is nonzero, (2.11) may be reformulated as follows. By (2.7), $\Omega-\kappa_{i}^{2}=-\kappa_{i}-\Lambda^{-1} \kappa_{i}^{-1}$. Substituting this expression into the third row of (2.11) and manipulating the determinant, we obtain

$$
\operatorname{det}\left(\begin{array}{ccc}
1 & 1 & 1 \\
e^{\kappa_{1} L} & e^{\kappa_{2} L} & e^{\kappa_{3} L} \\
\kappa_{1}^{-2} & \kappa_{2}^{-2} & \kappa_{3}^{-2}
\end{array}\right)=0
$$

Let us rule out possible multiple roots. First regarding a triple root, which by (2.8) must be $\kappa_{1}=\kappa_{2}=\kappa_{3}=\frac{1}{3}$. In this case the general solution to (2.6) is of the 
form $a(x)=\left(C_{0}+C_{1} x+C_{2} x^{2}\right) e^{x / 3}$ and the only possible eigenvalue is $\Omega=-\frac{1}{3}$ by (2.9). Substituting $a(x)$ and $\Omega$ into (2.6), we find there is no nontrivial solution and hence no eigenvalue. Now we assume $\kappa_{1}=\kappa_{2} \neq \kappa_{3}$ in $(2.8-2.10)$ and therefore the general solution to (2.6) is $a(x)=C_{1} e^{\kappa_{1} x}+C_{2} x e^{\kappa_{1} x}+C_{3} e^{\kappa_{3} x}$. Inserting $a(x)$ into the boundary conditions in $(2.6)$ and considering $(2.8-2.10)$ with $\kappa_{2}=\kappa_{1}$, we find the existence of a nontrivial solution requires $\kappa_{1}$ to satisfy both of the following equations

$$
\begin{aligned}
& \kappa_{1} L\left(4 \kappa_{1}^{2}-3 \kappa_{1}-1\right)+2\left(1-2 \kappa_{1}\right)^{2} \cdot\left[e^{\left(1-3 \kappa_{1}\right) L}-1\right]=0, \\
& -2 \kappa_{1}^{3}+\kappa_{1}^{2}=\Lambda^{-1}>0
\end{aligned}
$$

and the possible eigenvalue is then given by

$$
\Omega=3 \kappa_{1}^{2}-2 \kappa_{1} .
$$

Note that (2.13) only has isolated complex roots since its left hand side is a holomorphic function. Thus $(2.13-2.15)$ can only provide isolated eigenvalues in the $\Lambda^{-1}-\Omega$ plane and a perturbation of the parameter $\Lambda^{-1}$ will lead to the case when all $\kappa_{j}$ 's are different. So the case $\kappa_{1}=\kappa_{2} \neq \kappa_{3}$ can be obtained as limit of the case of distinct roots. In the analysis below we will see that the roots remain separated for $\Lambda^{-1}$ small or $L$ large.

3. Small Dispersion ${ }^{2}: \Lambda^{-1} \ll 1$. If $\Lambda^{-1}=0$, then $\mathscr{L}$ has eigenvalues

$$
\Omega_{0}^{(0)}=0,
$$

with the solution of $(2.8-2.10)$ given by $\kappa_{j}=0,0,1$, and

$$
\Omega_{n}^{(0)}=-\frac{1}{4}-\left(\frac{\pi n}{L}\right)^{2}, \quad n=1,2, \ldots
$$

with $\kappa_{j}=0, \frac{1}{2} \pm \frac{i n \pi}{L}$. We seek the first term in an expansion of $\Omega_{n}$ in powers of $\Lambda^{-1}$.

3.1. Perturbation of $\Omega_{n}, n \geq 1$. We prove that for $\Lambda^{-1}$ small,

$$
\Omega_{n}(\Lambda)=\Omega_{n}^{(0)}+\Omega_{n}^{(1)} \Lambda^{-1}+\mathcal{O}\left(\Lambda^{-2}\right)
$$

where $\Omega_{n}^{(0)}$ is given by $(3.2)$ and

$$
\Omega_{n}^{(1)}=-\frac{2}{1+4 \pi^{2} n^{2} L^{-2}}
$$

By the implicit function theorem, we may expand the solution of (2.8-2.10) and $(2.12)$ as

$$
\left\{\begin{array}{l}
\kappa_{1,2}=\frac{1}{2} \pm \frac{i n \pi}{L}+b_{1,2} \Lambda^{-1}+\mathcal{O}\left(\Lambda^{-2}\right) \\
\kappa_{3}=0+b_{3} \Lambda^{-1}+\mathcal{O}\left(\Lambda^{-2}\right) \\
\Omega_{n}=\Omega_{n}^{(0)}+\Omega_{n}^{(1)} \Lambda^{-1}+\mathcal{O}\left(\Lambda^{-2}\right)
\end{array}\right.
$$

\footnotetext{
${ }^{2}$ We remind the reader that in this section and the next, $\Lambda$ and $L$, without bars, refer to the dimensionless parameters defined by (2.3).
} 
Substituting into (2.8-2.10), we find from the $\mathcal{O}\left(\Lambda^{-1}\right)$ terms

$$
\left\{\begin{array}{l}
b_{1}+b_{2}+b_{3}=0 \\
\Omega_{n}^{(1)}=-\frac{b_{1}+b_{2}}{2}+\frac{i n \pi}{L}\left(b_{1}-b_{2}\right)-b_{3}, \\
b_{3}=\left(\frac{1}{4}+\frac{n^{2} \pi^{2}}{L^{2}}\right)^{-1} .
\end{array}\right.
$$

Substituting into (2.12) we find the leading order term $\mathcal{O}\left(\Lambda^{2}\right)$ vanishes identically; from the next order term $\mathcal{O}(\Lambda)$ we deduce that $b_{1}=b_{2}$. The relation (3.4) follows from this equation and (3.6).

3.2. Perturbation of $\Omega_{0}$. We prove that for $\Lambda^{-1}$ small,

$$
\Omega_{0}(\Lambda)=\Omega_{0}^{(1)} \Lambda^{-1}+\mathcal{O}\left(\Lambda^{-2}\right)
$$

where

$$
\Omega_{0}^{(1)}=-\left(1-\frac{L}{\exp (L)-1}\right)
$$

The expression analogous to (3.5) is complicated by the fact that, for $\Lambda^{-1}=0$, $\kappa_{1}=\kappa_{2}=0$ is a double root of $(2.8-2.10)$. Thus we seek a Puisseux expansion

$$
\left\{\begin{array}{l}
\kappa_{1,2}=a_{1,2} \Lambda^{-1 / 2}+b_{1,2} \Lambda^{-1}+\mathcal{O}\left(\Lambda^{-3 / 2}\right) \\
\kappa_{3}=1+b_{3} \Lambda^{-1}+\mathcal{O}\left(\Lambda^{-2}\right) \\
\Omega_{0}=\Omega_{0}^{(1)} \Lambda^{-1}+\mathcal{O}\left(\Lambda^{-2}\right)
\end{array}\right.
$$

First we substitute into (2.8-2.10); from the vanishing of the first two terms in the expansions we deduce

$$
\left\{\begin{array}{l}
a_{1}+a_{2}=0 \\
b_{1}+b_{2}+b_{3}=0 \\
\Omega_{0}^{(1)}=-a_{1} a_{2}-\left(b_{1}+b_{2}\right) \\
a_{1} a_{2}=1 \\
a_{1} b_{2}+a_{2} b_{1}=0
\end{array}\right.
$$

Note that the equation $a_{1}+a_{2}=0$ arises from the leading term of both (2.8) and (2.9). These equations imply that

$$
\left\{\begin{array}{l}
a_{1,2}= \pm i \\
b_{2}=b_{1} \\
b_{3}=-2 b_{1} \\
\Omega_{0}^{(1)}=-1-2 b_{1}
\end{array}\right.
$$

Then we substitute (3.11) into the determinant (2.11). The leading term in the resulting expansion, which is $\mathcal{O}\left(\Lambda^{-1}\right)$, vanishes. Requiring the $\mathcal{O}\left(\Lambda^{-3 / 2}\right)$ term to vanish yields the claim (3.8), even though we do not yet know the $b_{j}$ 's.

Incidentally, if desired, the $b_{j}$ 's may be determined by substituting (3.4) into (3.6), yielding

$$
b_{1}=b_{2}=-\frac{L}{2(\exp (L)-1)}=-\frac{b_{3}}{2} .
$$




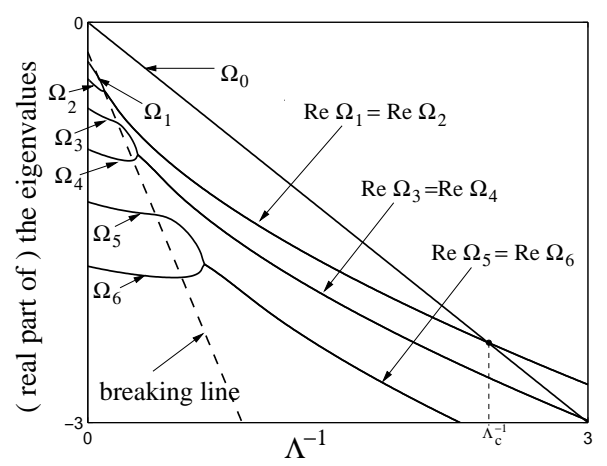

FIG. 4.1. The evolution of the real parts of the first seven eigenvalues $\Omega_{0}, \Omega_{1} \ldots \Omega_{6}$ vs. $\Lambda^{-1}$, assuming $L=15$. The dashed line is the breaking line described in (4.26), the estimate for where the eigenvalues $\Omega_{1}, \Omega_{2} \ldots$ become complex. $\Lambda_{c}^{-1}$ is the crossover point such that if $\Lambda^{-1}>\Lambda_{c}^{-1}$ the eigenvalue which has largest real part, $\Omega_{\max }$, is complex.

4. A Long Fiber $^{3}: L \gg 1$. To analyze larger values of $\Lambda^{-1}$, where the expansion of Section 3 loses accuracy, we need to require that $L \gg 1$. When $\Lambda^{-1}$ is small, all eigenvalues are real and ordered by their index: i.e.,

$$
\Omega_{0}>\Omega_{1}>\Omega_{2}>\ldots
$$

As $\Lambda^{-1}$ increases, some of the eigenvalues become complex. As this occurs we still retain the ordering

$$
\operatorname{Re} \Omega_{1} \geq \operatorname{Re} \Omega_{2}>\operatorname{Re} \Omega_{3} \geq \operatorname{Re} \Omega_{4}>\operatorname{Re} \Omega_{5} \ldots
$$

However, $\Omega_{0}$ remains real $^{4}$, and although its position in the sequence (4.2) varies with $\Lambda^{-1}$, we retain the index zero. This behavior is illustrated in Figure 4.1, where we have set $L=15$.

4.1. Computation of $\Omega_{0}$. We shall prove that, provided $L$ is sufficiently large, the operator $\mathscr{L}$ has a real eigenvalue

$$
\Omega_{0}=-\Lambda^{-1}+\mathcal{O}\left(e^{-L}\right) .
$$

Note that this result is consistent with (3.8). Recall from Section 3.2, that in solving (2.8-2.10) for small nonzero $\Lambda^{-1}$, we found $\kappa_{1,2}$ became a complex-conjugate pair while $\kappa_{3}$ remained real. Thus for general $\Lambda^{-1}$ we assume

$$
\kappa_{1,2}=\mu \pm i \nu
$$

Then (2.8) implies

$$
\kappa_{3}=1-2 \mu
$$

\footnotetext{
${ }^{3}$ We remind the reader that in this section, $\Lambda$ and $L$, without bars, refer to the dimensionless parameters defined by (2.3).

${ }^{4}$ This statement breaks down if $\Lambda^{-1}$ becomes exceedingly large, contradicting our implicit assumption that $\Lambda^{-1} \ll L$.
} 
We use (2.9-2.11) to solve for $\mu, \nu$ and $\Omega$ as follows. Dividing the middle row of (2.11) by $e^{\kappa_{3} L}$, we rewrite this equation as

$$
\operatorname{det}\left(\begin{array}{ccc}
\kappa_{1} & \kappa_{2} & \kappa_{3} \\
\kappa_{1} e^{\left(\kappa_{1}-\kappa_{3}\right) L} & \kappa_{2} e^{\left(\kappa_{2}-\kappa_{3}\right) L} & \kappa_{3} \\
\Omega_{0}-\kappa_{1}^{2} & \Omega_{0}-\kappa_{2}^{2} & \Omega_{0}-\kappa_{3}^{2}
\end{array}\right)=0
$$

Now provided

$$
\mu<\frac{1}{3}
$$

we have for the 2,1- and 2,2-entries of this determinant

$$
\left|e^{\left(\kappa_{j}-\kappa_{3}\right) L}\right|=e^{-(1-3 \mu) L} \ll 1,
$$

since $L \gg 1$. Thus neglecting these entries and recalling (4.4-4.5), we conclude

$$
\Omega_{0}=-\left(\mu^{2}+\nu^{2}\right)+\mathcal{O}\left(e^{-(1-3 \mu) L}\right) .
$$

On the other hand, we substitute (4.4-4.5) into (2.9) to find

$$
\Omega_{0}=-\left(\mu^{2}+\nu^{2}\right)-2 \mu(1-2 \mu) .
$$

Comparing (4.9) and (4.10), we deduce

$$
\mu(1-2 \mu)=\mathcal{O}\left(e^{-(1-3 \mu) L}\right) .
$$

By (4.7), we have $1-2 \mu>1 / 3>0$, so dividing (4.11) by $1-2 \mu$ we obtain

$$
\mu=\mathcal{O}\left(e^{-(1-3 \mu) L}\right)
$$

Thus assumption (4.7) is consistent and moreover

$$
\mu=\mathcal{O}\left(e^{-(1-3 \mu) L}\right)=\mathcal{O}\left(e^{-L}\right) .
$$

Returning to (4.9) we conclude that

$$
\Omega_{0}=-\nu^{2}+\mathcal{O}\left(e^{-L}\right) .
$$

But substituting (4.4-4.5) into (2.10) and working with (4.13), we see

$$
\Lambda^{-1}=\nu^{2}+\mathcal{O}\left(e^{-L}\right) .
$$

The claim (4.3) follows on eliminating $\nu$ from (4.14-4.15).

As one might expect with an exponentially small error, (4.3) agrees extremely well with the numerical results. Indeed the graph of (4.3) cannot be distinguished visually from the graph of $\Omega_{0}$ in Figure 4.1.

Incidentally, the eigenfunction associated with $\Omega_{0}$ exhibits unusual behavior for a one-dimensional eigenvalue problem - the number of its zeros or nodes changes as $\Lambda^{-1}$ varies. Specifically, modulo terms that are exponentially small (outside of a boundary layer near $x=L$ ) the nondimensionalized eigenfunction is just $\cos (x / \sqrt{\Lambda})$. 


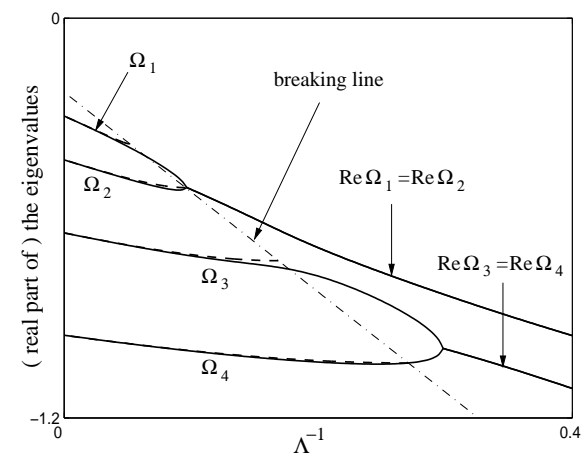

Fig. 4.2. A comparison between the computations (solid lines) and the theoretical approximations given by (4.24) (dashed lines) of the eigenvalues $\Omega_{1}, \ldots, \Omega_{4}$ while they are real. The approximation terminate at the breaking line, given by (4.26). The dimensionless cable length $L$ is 15.

4.2. Later eigenvalues, real case. In this subsection, we characterize a range of $\Lambda^{-1}$, as this parameter increases from 0 , in which the eigenvalue $\Omega_{n}$ is real. Continuing the structure of the roots $\kappa_{j}$ inherited from small $\Lambda^{-1}$, we assume

$$
\kappa_{1,2}=\mu \pm i \nu
$$

and invoke (2.8) to conclude

$$
\kappa_{3}=1-2 \mu \text {. }
$$

We shall solve (2.12) for $\nu$ and substitute the result into (2.9-2.10) to obtain a parametric representation of the curve $\Omega=\Omega_{n}\left(\Lambda^{-1}\right)$ in the $\Lambda^{-1}, \Omega--$ plane, with $\mu$ as the parameter. (It is not practical to solve explicitly for $\Omega_{n}$ as a function of $\Lambda^{-1}$.)

Let us divide the second row of the determinant $(2.12)$ by $\exp (\mu L)$, obtaining

$$
\operatorname{det}\left(\begin{array}{ccc}
1 & 1 & 1 \\
e^{i \nu L} & e^{-i \nu L} & e^{(1-3 \mu) L} \\
\kappa_{1}^{-2} & \kappa_{2}^{-2} & \kappa_{3}^{-2}
\end{array}\right)=0 .
$$

If

$$
\mu>\frac{1}{3}
$$

then for large $L$ we may neglect the $2,3-$-entry of this determinant, so that the equation reduces to

$$
e^{2 i \nu L}=\frac{\kappa_{2}^{2}\left(\kappa_{1}^{2}-\kappa_{3}^{2}\right)}{\kappa_{1}^{2}\left(\kappa_{2}^{2}-\kappa_{3}^{2}\right)}+\mathcal{O}\left(e^{-(3 \mu-1) L}\right) .
$$

Equation (4.20) suggests that $\nu L=\mathcal{O}(1)$ or

$$
\nu=\mathcal{O}\left(L^{-1}\right)
$$

Assuming this and recalling (4.19), we see that the right hand side of (4.20) equals $1+\mathcal{O}\left(L^{-1}\right)$. Solving $(4.20)$ we find

$$
\nu=n \pi \cdot L^{-1}+\mathcal{O}\left(L^{-2}\right), \quad n=1,2, \ldots
$$


confirming our assumption (4.21). On the other hand, substituting (4.16-4.17) into $(2.8-2.9)$ we find

$$
\left\{\begin{array}{l}
\Lambda^{-1}=(1-2 \mu)\left(\mu^{2}+\nu^{2}\right) \\
\Omega_{n}=3 \mu^{2}-2 \mu-\nu^{2}
\end{array}\right.
$$

Substituting (4.22) into (4.23) we obtain the parametric representation

$$
\left\{\begin{array}{l}
\Lambda^{-1}=(1-2 \mu) \cdot\left(\mu^{2}+n^{2} \pi^{2} L^{-2}\right)+\mathcal{O}\left(L^{-3}\right), \\
\Omega_{n}=3 \mu^{2}-2 \mu-n^{2} \pi^{2} L^{-2}+\mathcal{O}\left(L^{-3}\right) .
\end{array}\right.
$$

Note from (4.24) that $\Lambda^{-1}=0$ occurs when $\mu=1 / 2$. (This may also be seen by solving (2.8-2.11) directly when $\Lambda^{-1}=0$, which avoids the $\mathcal{O}\left(L^{-3}\right)$ error in (4.24).) Recalling (4.19), we conclude that

$$
1 / 3<\mu \leq 1 / 2
$$

is the relevant parameter range in (4.24). At the lower end of the range, the points $\left(\Lambda^{-1}, \Omega\right)$ in $(4.24)$ all lies along the line

$$
\Omega=-3 \Lambda^{-1}-2 / 9
$$

which we call the breaking line. This may be seen, avoiding the $\mathcal{O}\left(L^{-3}\right)$-errors, by setting $\mu=1 / 3$ in (4.23) and eliminating $\nu^{2}$.

The approximations (4.24), for $n=1,2,3,4$ and for $\mu$ satisfying (4.25) are graphed in Figure 4.2, along with the computed eigenvalues. As the figure emphasizes, the asymptotics underlying (4.24) break down as $\mu \rightarrow 1 / 3$. More precisely, for $\mu$ near $1 / 3$, exceedingly large values of $L$ are needed to make (4.24) accurate, and increasingly so as $n$ becomes large.

Incidentally, note that for $\Lambda^{-1}=0$,

$$
\frac{d \Omega_{n}}{d \Lambda^{-1}}=\left.\frac{d \Omega_{n} / d \mu}{d \Lambda^{-1} / d \mu}\right|_{\mu=\frac{1}{2}}=-\frac{2}{1+4 n^{2} \pi^{2} L^{-2}},
$$

which is consistent with (3.4), even without the $\mathcal{O}\left(L^{-3}\right)$-errors in (4.24).

4.3. Later eigenvalues, complex case. Motivated by the numerical results, when the above asymptotics break down we look for complex eigenvalues. Let us define

$$
\begin{aligned}
\mu & =\frac{1}{2} \operatorname{Re}\left(\kappa_{1}+\kappa_{2}\right), \\
\nu & =\frac{1}{2} \operatorname{Im}\left(\kappa_{1}+\kappa_{2}\right) .
\end{aligned}
$$

Then

$$
\kappa_{1,2}=\mu+i \nu \pm \delta
$$

where $\delta$, possibly complex, is to be determined, and (2.8) implies that

$$
\kappa_{3}=1-2(\mu+i \nu)
$$




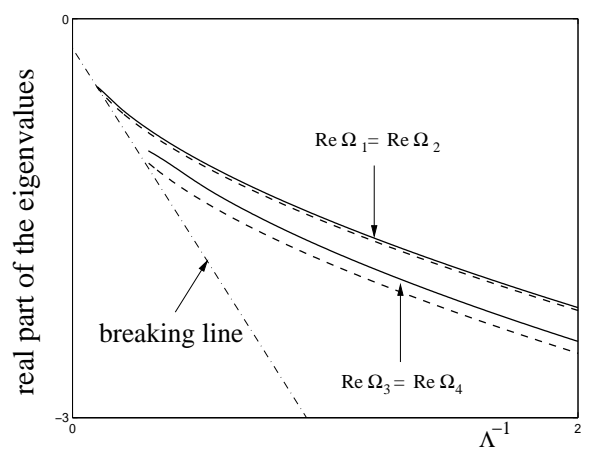

FIG. 4.3. A comparison between the computations (solid lines) and the theoretical approximations given by $(4.36,4.40)$ (dashed lines) of the real parts of the eigenvalues $\Omega_{1}, \ldots \Omega_{4}$ while they are complex. The dimensionless cable length $L$ is 15 .

Even in complex case, we continue to assume (4.19). Thus, asymptotically for large $L$, the determinant equation (2.12) may be simplified to

$$
e^{2 \delta L}=\frac{\kappa_{2}^{2}\left(\kappa_{1}^{2}-\kappa_{3}^{2}\right)}{\kappa_{1}^{2}\left(\kappa_{2}^{2}-\kappa_{3}^{2}\right)}+\mathcal{O}\left(e^{-(3 \mu-1) L}\right) .
$$

As above, we solve (4.32) to obtain

$$
\delta=i n \pi \cdot L^{-1}+\mathcal{O}\left(L^{-2}\right), \quad n=1,2, \ldots
$$

We determine the real parameter $\nu$ from the condition that $\Lambda^{-1}$ must be real: i.e., by (2.10)

$$
\operatorname{Im}\left(\kappa_{1} \kappa_{2} \kappa_{3}\right)=0,
$$

and from this we deduce that

$$
\nu= \pm\left\{\mu(3 \mu-1)+n^{2} \pi^{2} L^{-2}+\mathcal{O}\left(L^{-3}\right)\right\}^{1 / 2} .
$$

Substituting into (2.9-2.10) we obtain a parametric representation of $\Omega$ versus $\Lambda^{-1}$. Specifically, adjusting indices to account for the fact that (4.35) has two solutions, we find

$$
\begin{aligned}
& \Lambda^{-1}=\mu(4 \mu-1)^{2}+4 \mu \cdot n^{2} \pi^{2} L^{-2}+\mathcal{O}\left(L^{-3}\right), \\
& \Omega_{2 n}=(\mu+i \nu)(3 \mu+3 i \nu-2)-n^{2} \pi^{2} L^{-2}+\mathcal{O}\left(L^{-3}\right), \\
& \Omega_{2 n-1}=\bar{\Omega}_{2 n},
\end{aligned}
$$

where $\nu$ is given by $(4.35)$ and

$$
1 / 3<\mu<\infty .
$$

By substituting $\mu=1 / 3$ into $(4.36,4.37)$ we see that these approximations terminate at the breaking line (4.26). Several of them are graphed in Figure 4.3, along with the 
computed eigenvalues. Incidentally, note that the real parts of the eigenvalues may be written more simply, without $\nu$,

$$
\operatorname{Re} \Omega_{2 n-1}=\operatorname{Re} \Omega_{2 n}=-\mu(6 \mu-1)-4 n^{2} \pi^{2} L^{-2}+\mathcal{O}\left(L^{-3}\right) .
$$

To explore the transition between the real and complex cases, we substitute the limiting value $\mu=1 / 3$ into $(4.24)$ and $(4.36,4.37)$. For the even-index eigenvalues, both the real and complex cases give same result,

$$
\begin{aligned}
& \Lambda^{-1}=\frac{1}{27}+\frac{1}{3}\left(\frac{2 n \pi}{L}\right)^{2}+\mathcal{O}\left(L^{-3}\right), \\
& \Omega_{2 n}=-\frac{1}{3}-\left(\frac{2 n \pi}{L}\right)^{2}+\mathcal{O}\left(L^{-3}\right)
\end{aligned}
$$

which of course lies on the breaking line (4.26). For odd-index eigenvalues, there is an $\mathcal{O}\left(L^{-2}\right)$ jump between the results of substituting $\mu=1 / 3$ into $(4.24)$ and $(4.36,4.37)$. Hints of this behavior may be seen in Figure 4.2 - the asymptotic approximation of $\Omega_{2 n}$ in the real case continues to be defined closer to the actual transition to complex eigenvalues than that of $\Omega_{2 n-1}$. Incidentally, (4.41) may be used to estimate the largest value of $\Lambda^{-1}$ at which $\Omega_{2 n-1}$ and $\Omega_{2 n}$ are still real.

Let us relate these formulae to a result of Echebarria and Karma [6]. Invoking considerations of group velocity and bifurcation theory, those authors argue that, at the onset of instability the complex wave number $(\kappa=\mu+i \nu$ in our notation) ought to satisfy

$$
2 \kappa^{3}-\kappa^{2}+\frac{1}{\Lambda}=0
$$

(This relation is equivalent to their equation (56) written in our notation.) Now to leading order equations $(4.35,4.36)$ assert that

$$
\nu^{2}=\mu(3 \mu-1), \quad 16 \mu^{3}-8 \mu^{2}+\mu=\Lambda^{-1} .
$$

It may be checked that these two real equations are equivalent to the single complex equation (4.43). Thus we have given an independent derivation of (4.43) that does not rely on computing group velocities for waves that grow exponentially in space and that accounts explicitly for boundary conditions on a (long) finite-length cable.

5. Discussion and conclusion. Let us summarize the asymptotic results from Section 4 regarding the eigenvalues of the linear operator of (1.4), which is $\bar{w}^{2} \mathscr{L}=$ $\left(w^{2} / \xi^{2}\right) \cdot \mathscr{L}$. For this task, and for the remainder of the paper, we shall undo the scaling (2.1) and (2.3), and return to the dimensional parameters. We found

- $\Omega_{0}$ is always real given by

$$
\Omega_{0}=-\frac{\xi^{2}}{w \Lambda}+\mathcal{O}\left(e^{-w L / \xi^{2}}\right)
$$

- For $n \geq 1, \Omega_{n}$ is real if $\Lambda^{-1}$ is below a threshold. Equation (4.41) estimates that the threshold for $\Omega_{2 n-1}$ and $\Omega_{2 n}$ to become complex is

$$
\frac{w^{3}}{27 \xi^{4}}+\frac{w}{3}\left(\frac{2 n \pi}{L}\right)^{2}+\mathcal{O}\left(L^{-3}\right) .
$$




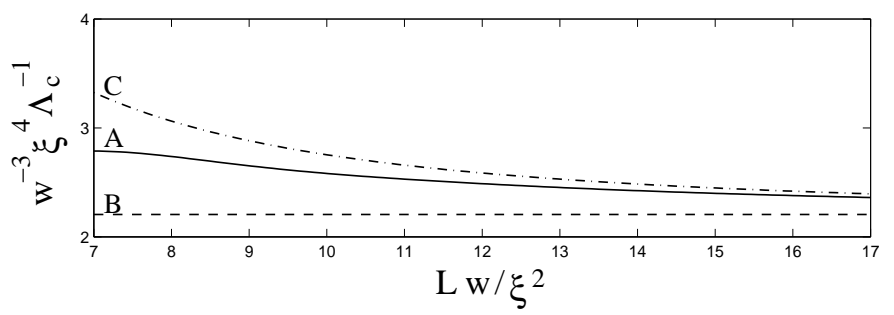

FIG. 5.1. A comparison among the computational result (curve $A$ ) for $\Lambda_{c}^{-1}$, the theoretical leading order approximation (5.6) (line B) and the second order approximation (5.7) (curve C). The $x$-axis is the cable length $L$ scaled by $w / \xi^{2}$ and $y$-axis is the critical value $\Lambda_{c}^{-1}$ scaled by $w^{-3} \xi^{4}$. With these scalings, the curves are independent of $\xi$ and $w$.

- In the real case, the relation between $\Lambda^{-1}$ and $\Omega_{n}$ is given parametrically by

$$
\left\{\begin{array}{l}
\Lambda^{-1}=\xi^{-2}(w-2 \mu \xi) \cdot\left(\mu^{2}+n^{2} \pi^{2} \xi^{2} L^{-2}\right)+\mathcal{O}\left(L^{-3}\right) \\
\Omega_{n}=3 \mu^{2}-2 \mu w / \xi-n^{2} \pi^{2} \xi^{2} L^{-2}+\mathcal{O}\left(L^{-3}\right)
\end{array}\right.
$$

where $w / 3 \xi<\mu \leq w / 2 \xi$.

- In the complex case, the relation between $\Lambda^{-1}$ and real parts of $\Omega_{2 n-1}, \Omega_{2 n}$ is given parametrically by

$$
\left\{\begin{array}{l}
\Lambda^{-1}=\mu \xi^{-1}(4 \mu-w / \xi)^{2}+4 \mu \xi \cdot n^{2} \pi^{2} L^{-2}+\mathcal{O}\left(L^{-3}\right), \\
\operatorname{Re} \Omega_{2 n-1}=\operatorname{Re} \Omega_{2 n}=-\mu(6 \mu-w / \xi)-4 n^{2} \pi^{2} \xi^{2} L^{-2}+\mathcal{O}\left(L^{-3}\right), \\
\operatorname{Im} \Omega_{2 n-1}=-\operatorname{Im} \Omega_{2 n}=2(3 \mu-w / \xi)\left[\mu(3 \mu-w / \xi)+n^{2} \pi^{2} \xi^{2} L^{-2}\right]^{1 / 2}+\mathcal{O}\left(L^{-3}\right),
\end{array}\right.
$$

where $w / 3 \xi<\mu<\infty$.

Consider bifurcation of (1.2) from the zero solution as $\sigma$ increases. An eigenvalue of the linearization crosses into the (unstable) right-half-plane when $\sigma=\operatorname{Re} \Omega_{\max }$ where $\Omega_{\max }$ is the eigenvalue of (1.4) with the (algebraically) largest real part. If $\Lambda^{-1}$ is small, $\Omega_{0}$ is the largest eigenvalue, its associated eigenfunction is real, and a timeindependent, stationary-wave solution of (1.2) appears at the bifurcation. However, $\Omega_{0}$ decreases more rapidly with $\Lambda^{-1}$ than later eigenvalues, which moreover become complex. Thus, when $\Lambda^{-1}$ is sufficiently large, say $\Lambda^{-1}>\Lambda_{c}^{-1}, \Omega_{\max }$ will be complex, and time-oscillatory, travelling-wave solutions will appear at the bifurcation (see [6] for more details). To estimate the crossover value $\Lambda_{c}^{-1}$, we consider the equation $\Omega_{0}=\operatorname{Re} \Omega_{1}$. Recalling (5.1), we may rewrite this equation, to leading order as

$$
-\frac{\xi^{2}}{w \Lambda}=\operatorname{Re} \Omega_{1}
$$

Substituting the first and second equation of (5.4) on the left and right of (5.5), respectively, we obtain the quadratic equation

$$
8 \mu^{2}-7(w / \xi) \cdot \mu+(w / \xi)^{2}=0
$$

for the value of $\mu$ associated with the crossover. We select the root $\mu=(7+\sqrt{17}) w / 16 \xi$ that satisfies $\mu>w / 3$ and substitute into (4.36) to obtain the leading-order estimate

$$
\Lambda_{c}^{-1} \approx \frac{71+17 \sqrt{17}}{64} \cdot \frac{w^{3}}{\xi^{4}} .
$$


By carrying $\mathcal{O}\left(L^{-2}\right)$ terms in the above calculation, one may extend this estimate to next order

$$
\Lambda_{c}^{-1}=\frac{w^{3}}{\xi^{4}}\left\{\frac{71+17 \sqrt{17}}{64}+\frac{(7+\sqrt{17}) \pi^{2}}{2} \cdot\left(\frac{w L}{\xi^{2}}\right)^{-2}\right\}+\mathcal{O}\left(L^{-3}\right) .
$$

Figure 5.1 shows a comparison between the computational result for $\Lambda_{c}^{-1}$ and theoretical approximations (5.6) and (5.7) for various (large) cable length $L$. Observe that the computational value is between the values of (5.6) and (5.7). For $L$ smaller than shown in the figure, the graph of $\Lambda_{c}^{-1}$ changes character. A hint of such behavior may be gleaned from the fact that the approximation (5.7) blows up as $L^{-2}$ as $L$ tends to zero. We plan to investigate these phenomena more thoroughly in a future publication.

Table 5.1 summarizes the results of two simulations that illustrate the different behavior that occurs for $\Lambda^{-1}>\Lambda_{c}^{-1}$ and $\Lambda^{-1}<\Lambda_{c}^{-1}$. The two-current model [7], a simplified cardiac model similar in spirit to FitzHugh-Nagumo model, is discussed in the appendix of this paper. The Noble model [8] was an early attempt to adapt a Hodgkin-Huxley-type model to cardiac cells. Because realism was attempted, this model is substantially more complicated than the two-current model. The key behavior relevant here is that the conduction-velocity curve is exceptionally flat at the critical diastolic interval, which makes $\Lambda^{-1}=2 c^{\prime} / c^{2}$ small [5]. Simulations with the Noble model are presented in [6].

TABLE 5.1

Results of two simulations: Two-current model and Noble's model. $w, \xi$, L have units of length in $\mathrm{cm} ; \Lambda$ and $\Lambda_{c}$ have units of inverse length in $\mathrm{cm}^{-1}$.

\begin{tabular}{|c|c|c|c|c|c|c|}
\hline Name of model & $w$ & $\xi$ & $L$ & $\Lambda^{-1}$ & $\Lambda_{c}^{-1}$ & Observed alternans \\
\hline \hline Two-current & 0.034 & 0.310 & 25 & 0.206 & 0.011 & Traveling \\
\hline Noble & 0.045 & 0.180 & 20 & 0.020 & 0.198 & Stationary \\
\hline
\end{tabular}

Appendix: Alternans. In this appendix we illustrate the phenomena of alternans in the context of a simple cardiac model [7], which is similar in spirit to the FitzHugh-Nagumo equation. A single heart cell in this model is described by two dimensionless functions of time, a scaled voltage $v$ and a gate $h$ that satisfy a set of ODE's

$$
\frac{d v}{d t}=J_{\text {ion }}(v, h)+J_{\text {stim }}(t)
$$

where the ionic current

$$
J_{\text {ion }}(v, h)=\frac{h}{\tau_{\text {in }}} v^{2}(1-v)-\frac{v}{\tau_{\text {out }}}
$$

and $J_{\text {stim }}(t)$ is an external current applied repeatedly in brief pulses (cf. (A.4) below), and

$$
\frac{d h}{d t}= \begin{cases}-\frac{h}{\tau_{\text {close }}} & \text { if } v>v_{\text {crit }} \\ \frac{1-h}{\tau_{\text {open }}} & \text { if } v<v_{\text {crit }}\end{cases}
$$


TABLE 5.2

Representative values for the parameters in the two-current model.

\begin{tabular}{|c|c|c|c|c|c|c|}
\hline$\tau_{\text {in }}$ & $\tau_{\text {out }}$ & $\tau_{\text {open }}$ & $\tau_{\text {close }}$ & $v_{\text {crit }}$ & $K$ & $L$ \\
\hline $0.2 \mathrm{~ms}$ & $7 \mathrm{~ms}$ & $50 \mathrm{~ms}$ & $130 \mathrm{~ms}$ & 0.05 & $0.4 \mathrm{~cm}^{2} \cdot \mathrm{s}^{-1}$ & $25 \mathrm{~cm}$ \\
\hline
\end{tabular}

Representative values for the parameters in these equations are given in Table 5.2, note that $K$ is the diffusion coefficient that appears in the PDE (A.11).

In the absence of a stimulus current, i.e. $J_{\text {stim }}=0,($ A.1-A.3) have a stable equilibrium at $(v, h)=(0,1)$. Suppose this equilibrium is perturbed by a sequence of stimuli, applied with period $B$ (mnemonic for basic cycle length), say

$$
J_{\text {stim }}(t)=\left\{\begin{array}{l}
v_{\text {stim }} / \delta \quad \text { if } 0<t<\delta \quad(\bmod B), \\
0 \quad \text { otherwise }
\end{array}\right.
$$

where $\delta \ll \tau_{\text {in }}$ and $v_{\text {stim }}$ is not excessively small. Provided this pacing is not too rapid, the stimuli produce action potentials as illustrated in Figure 5.2: i.e. each stimulus, although very brief, triggers an extended rise in the voltage, after which the voltage decays. Let the action potential duration (acronym APD; in mathematical formulae $A_{n}$ ) and diastolic interval (acronym DI; in mathematical formulae $D_{n}$ ) be defined as in the figure; note that $A_{n}+D_{n}=B$, where $B$ is the period or basic cycle length.

In [7] it is shown that, under the following assumption,

$$
\tau_{\text {in }} \ll \tau_{\text {out }} \ll \tau_{\text {open }}, \tau_{\text {close }}
$$

these variables approximately satisfy

$$
A_{n+1}=F\left(D_{n}\right)
$$

where

$$
F\left(D_{n}\right)=\tau_{\text {close }} \ln \left\{\frac{1-\left(1-h_{\min }\right) e^{-D_{n} / \tau_{\text {open }}}}{h_{\min }}\right\} .
$$

with $h_{\min }=4 \tau_{\text {in }} / \tau_{\text {out }}$. Since $D_{n}=B-A_{n}$, the sequence $A_{n}$ is determined by iteration of a 1D-map,

$$
A_{n+1}=F\left(B-A_{n}\right) .
$$

Provided $B$ is not too small, a sequence generated by (A.8) converges to a stable fixed point $A_{*}(B)$. However, for $B$ smaller than some critical value, $B_{\text {crit }}$, the fixed point loses its stability and we have a period doubling bifurcation of $A_{n}$, to a response called alternans. Let $A_{\text {crit }}$ be the fixed point solution to (A.8) for $B=B_{\text {crit }}$, and define $D_{\text {crit }}=B_{\text {crit }}-A_{\text {crit }}$. Recognizing that $\left|F^{\prime}\left(D_{\text {crit }}\right)\right|=1$ is the condition for bifurcation, we find

$$
D_{\text {crit }}=\tau_{\text {open }} \ln \left\{\left(1-h_{\text {min }}\right)\left(1+\frac{\tau_{\text {close }}}{\tau_{\text {open }}}\right)\right\},
$$

and thus

$$
A_{\text {crit }}=F\left(D_{\text {crit }}\right)=\tau_{\text {close }} \ln \left\{\frac{\tau_{\text {close }}}{\left(\tau_{\text {open }}+\tau_{\text {close }}\right) h_{\text {min }}}\right\}
$$




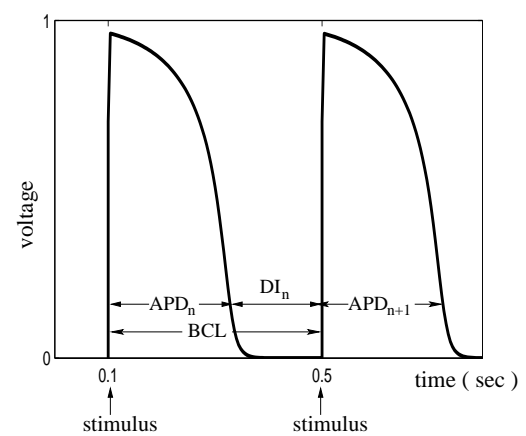

FIG. 5.2. An illustration of the solution to ODE system (A.1-A.3) with parameters given in the Table 5.2, assuming a periodic stimulus with period $\mathrm{BCL}=400 \mathrm{~ms}$.

In a homogenized cardiac fiber, say $0<x<L$, (A.1) is augmented by a diffusion term to obtain a PDE

$$
\partial_{t} v=K \partial_{x x} v+J_{\text {ion }}(v, h)+J_{\text {stim }}(x, t)
$$

equation (A.3) does not acquire any additional terms, but the $t$-derivative must be reinterpreted as a partial derivative. The stimulus current is applied locally near one end of the fiber and vanishes elsewhere. No-flux boundary conditions are imposed at both ends of the fiber:

$$
\partial_{x} v(0, t)=\partial_{x} v(L, t)=0 .
$$

The action potentials stimulated near $x=0$ propagate along the fiber. The travelling speed $c$, or the conduction velocity $(\mathrm{CV})$ of a periodic wave train, depends on the diastolic interval $D$,

$$
c=c(D) \approx \sqrt{\frac{K \cdot h_{\text {init }}}{2 \tau_{\text {in }}}}\left(1-\frac{3 h_{\mathrm{min}}}{4 h_{\mathrm{init}}}\right)
$$

where

$$
h_{\text {init }}(D)=1-\left(1-h_{\min }\right) e^{-D / \tau_{\text {open }}} .
$$

If the BCL of the stimuli is sufficiently small (i.e., if the pacing frequency is high enough), alternans will appear along the cable.

Let $A_{k}(x)$ denote the duration of the $k^{\text {th }}$ action potential at position $x$. Assuming $A_{k}(x)$ has the form (1.1) in Section 1. Echebarria and Karma[6] derived the approximate equation (1.2) to describe the evolution of the amplitude of alternans. The parameters $\Lambda, w, \xi$ that appear in (1.2) are estimated by

$$
\left\{\begin{array}{l}
\Lambda^{-1}=c^{\prime} / c^{2}, \\
w=2 K / c \\
\xi=\sqrt{K \cdot A_{\text {crit }}},
\end{array}\right.
$$

where $c$ and $c^{\prime}=\frac{d c}{d D}$ in (A.15) are both evaluated at the critical value $D=D_{\text {crit }}$. Note that $D_{\text {crit }}$ and $A_{\text {crit }}$ are given by $\left(\right.$ A.9, A.10). Evaluating $c\left(D_{\text {crit }}\right)$ by substituting 


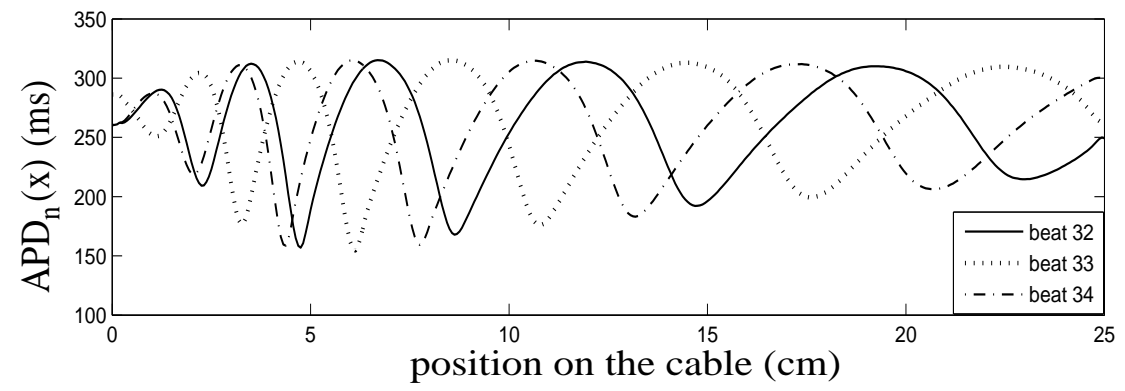

FIG. 5.3. Simulation of the PDE (A.11) with parameters given in the Table 5.2, assuming a periodic stimulus with period $\mathrm{BCL}=343 \mathrm{~ms}$. The $x$-axis is the position on the cable, the $y$-axis is $A_{n}(x)$ for the values of $n$ listed above. As predicted by using (5.7), the pattern is propagating.

the parameters of Table 5.2 into (A.13), we compute $w$ and $\xi$ as given in Table 5.1. On the other hand by differentiating (A.13) we obtain

$$
c^{\prime}=\frac{d c}{d D}=\frac{1-h_{\min }}{\tau_{\text {open }}} \sqrt{\frac{K}{2 \tau_{\text {in }}}} \cdot\left(\frac{1}{2} h_{\text {init }}^{-1 / 2}+\frac{3}{8} h_{\text {min }} h_{\text {init }}^{-3 / 2}\right) e^{-D / \tau_{\text {open }}},
$$

and after substituting we find $\Lambda^{-1}$ as given in Table 5.1. The simulation shown in Figure 5.3 were performed on a cable of length $25 \mathrm{~cm}$, which corresponds to a scaled, dimensionless length

$$
\overline{\bar{L}}=w L / \xi^{2}=8.84
$$

For this length, the (dimensionless) critical value $\Lambda_{c}^{-1}$ (computed numerically — curve $A$ in Figure 5.1) equals 2.67, which in dimensional units gives the value listed in Table 5.1. Since $\Lambda^{-1}>\Lambda_{c}^{-1}$, the modulation equation predicts that alternans will appear in travelling patterns. The behavior is observed in the simulation of Figure 5.3, which shows $A_{n}(x)$ for several beats about halfway through the transient to steady state.

\section{REFERENCES}

[1] R. F. Gilmour JR. And D. R. Chialvo, Electrical restitution, Critical mass, and the riddle of fibrillation, J. Cardiovasc. Electrophysiol., 10(1999), 1087-1089.

[2] A. Garfinkel et al., Preventing Ventricular Fibrillation by Flattening Cardiac Restitution, Proc. Natl. Acad. Sci. USA 97(2000), 6061-6066.

[3] A. V. PAnfilov, Spiral breakup as a model of ventricular fibrillation, Chaos 8(1998), 57-64.

[4] D. R. Chinlvo, D. C. Michaels and J. Jalife, Supernormal excitability as a mechanism of chaotic dynamics of activation in cardiac Purkinje fibers, Circ. Res. 66(1990) 525-545.

[5] B. EChEBARRIA AND A. KARMA, Instability and spatiotemporal dynamics of alternans in paced cardiac tissue, Phys. Rev. Lett. 88(2002) 208101.

[6] - Amplitude-equation approach to spatiotemporal dynamics of cardiac alternans, Phys. Rev. E 76(2007), 051911.

[7] C. C. Mitchell and D. G. Schaeffer, A two-current model for the dynamics of the cardiac membrane, Bull. Math. Bio. 65(2003) 767-793.

[8] D. Noble, A modification of the Hodgkin-Huxley equations applicable to Purkinje fibre action and pace-maker potentials, J. Physiol 160(1962) 317-352.

[9] http://www.americanheart.org/

[10] http://www.hrspatients.org/patients/heart_disorders/cardiac_arrest/ 